\title{
On-farm colorimetric detection of Pasteurella multocida, Mannheimia haemolytica, and Histophilus somni in crude bovine nasal samples
}

\author{
Ana Pascual-Garrigos 1,2,3, Murali Kannan Maruthamuthu1,3, Aaron Ault ${ }^{4}$, Josiah Levi Davidson 1,3, \\ Grigorii Rudakov ${ }^{1,3,5}$, Deepti Pillai ${ }^{6}$, Jennifer Koziol ${ }^{7}$, Jon P. Schoonmaker ${ }^{8}$, Timothy Johnson ${ }^{8}$ and \\ Mohit S. Verma ${ }^{1,3,5^{*}}$ (1)
}

\begin{abstract}
This work modifies a loop-mediated isothermal amplification (LAMP) assay to detect the bovine respiratory disease (BRD) bacterial pathogens Pasteurella multocida, Mannheimia haemolytica, and Histophilus somni in a colorimetric format on a farm. BRD causes a significant health and economic burden worldwide that partially stems from the challenges involved in determining the pathogens causing the disease. Methods such as polymerase chain reaction (PCR) have the potential to identify the causative pathogens but require lab equipment and extensive sample processing making the process lengthy and expensive. To combat this limitation, LAMP allows accurate pathogen detection in unprocessed samples by the naked eye allowing for potentially faster and more precise diagnostics on the farm. The assay developed here offers 66.7-100\% analytical sensitivity, and 100\% analytical specificity (using contrived samples) while providing $60-100 \%$ concordance with PCR results when tested on five steers in a feedlot. The use of a consumer-grade water bath enabled on-farm execution by collecting a nasal swab from cattle and provided a colorimetric result within $60 \mathrm{~min}$. Such an assay holds the potential to provide rapid pen-side diagnostics to cattle producers and veterinarians.
\end{abstract}

Keywords: diagnostics, bovine respiratory disease, loop-mediated isothermal amplification, pen-side

\section{Introduction}

Bovine respiratory disease (BRD) is known to be the most common cause of morbidity and mortality in cattle affecting $16 \%$ of all beef cattle [1] and causing up to $75 \%$ feedlot morbidity in North America [2]. It is also estimated to cost up to $\$ 900$ million annually in the beef industry alone [3]. This economic burden includes animal weight loss, labor expenses, pharmaceutical costs, and

*Correspondence: msverma@purdue.edu

${ }^{1}$ Department of Agricultural and Biological Engineering, Purdue University, 225 S University Street, West Lafayette, IN 47907, USA

Full list of author information is available at the end of the article deaths [4]. Rapid on-farm diagnostics have the potential to reduce this economic burden.

BRD is an umbrella term used to describe a condition caused by bacteria, viruses, or co-infection [5, 6]. Generally, it is detected by observing clinical signs such as nasal discharge, depression, anorexia, cough, and fever [4]. However, these clinical signs are insufficient for determining the underlying causative pathogen. Currently, one method for determining which pathogen is causing BRD involves taking a nasal swab sample from the suspected animal and sending it to a diagnostic laboratory, where tests are carried out to identify pathogens [7]. original author(s) and the source, provide a link to the Creative Commons licence, and indicate if changes were made. The images or other third party material in this article are included in the article's Creative Commons licence, unless indicated otherwise in a credit line to the material. If material is not included in the article's Creative Commons licence and your intended use is not permitted by statutory regulation or exceeds the permitted use, you will need to obtain permission directly from the copyright holder. To view a copy of this licence, visit http://creativecommons.org/licenses/by/4.0/. The Creative Commons Public Domain Dedication waiver (http://creativeco mmons.org/publicdomain/zero/1.0/) applies to the data made available in this article, unless otherwise stated in a credit line to the data. 
Some of the existing methods used for identifying the pathogen include culturing, enzyme-linked immunosorbent assays (ELISA), electron microscopy, immunohistochemistry, microarrays, and quantitative PCR (qPCR) detection [7, 8]. Unfortunately, tests of this nature often require specialized equipment, extensive sample preparation, and trained scientists making the process costly and lengthy. A mainstay of clinical therapy is the use of broad-spectrum antibiotics, which can have high failure rates [9]. Antibiotic therapy can fail for several reasons including misdiagnosis, inappropriate drug selection, inappropriate administration rate, dehydration, etc. At the same time, the use of antibiotics is claimed to be the leading cause of antimicrobial resistance, which makes the problem worse over time [10]. Thus, diagnostics that identify the pathogen and potentially their antibiotic resistance can help improve treatment by guiding towards targeted antibiotic use.

Unlike the lab-based methods mentioned above, isothermal DNA amplification methods such as recombinase polymerase amplification (RPA) [11] and loop-mediated isothermal amplification (LAMP) [1214] have recently been shown to accurately detect BRD pathogens directly from nasopharyngeal or nasal swabs used on cattle $[15,16]$. Like the versatile PCR, LAMP can detect sections of DNA found in infectious pathogens. However, it can do so in under an hour and without the need of a thermal cycler [17] making it more fieldfriendly for use in non-lab spaces such as farms.

We have recently designed a LAMP assay to detect the presence of Pasteurella multocida, Mannheimia haemolytica, and Histophilus somni from bovine nasal samples using a fluorescence reporter with $89 \%$ analytical specificity and $99 \%$ analytical sensitivity [16]. In the current work, we report a colorimetric assay that can be conducted on the farm to detect these bacterial pathogens. The colorimetric assay has a limit of detection of 1250 copies of DNA per reaction, an analytical specificity of $100 \%$, and an analytical sensitivity in the range of 66.7-100\% (when measured using contrived samples). The color change is visible to the naked eye and quantifiable using a camera. We illustrate the functionality of this assay on a feedlot by using a simple water bath based on consumer-grade precision cookers and conducting the assay on nasal samples obtained from five steers. The onfarm results are in $60-100 \%$ agreement with PCR assays conducted in the lab on the same samples.

\section{Materials and methods}

\section{Bacterial isolates and mucus sample collection}

Glycerol stocks of P. multocida, M. haemolytica, and H. somni isolates were obtained from Purdue University's Indiana Animal Disease Diagnostic Laboratory
(ADDL), as previously described [16]. These isolates were originally cultured by ADDL as a part of routine diagnostic testing from lung/nasopharyngeal sample submissions and identified using matrix-assisted laser desorption/ionization time-of-flight mass spectrometry (MALDI-TOF MS) analysis. Mucus samples were obtained from steers $(n=5)$ approximately 12 months of age and 600 lbs. in weight that had not been given antibiotics for at least 100 days at a feedlot in Indiana (Purdue Animal Care and Use Committee Approval \#1906001911) using rayon-tipped sterile double swabs designed for general specimen laboratory use (BD 220135, Becton, Dickinson, and Company, Franklin Lakes, NJ, USA). Each animal was restrained in a livestock handling chute and the animal head was restrained to minimize movement. The nostrils were wiped with paper towels to remove excess mucus. One double swab was inserted into both nostrils sequentially at a depth of approximately $5 \mathrm{~cm}$. The swabs were swirled in tubes with $200 \mu \mathrm{L}$ of DNA-free water and aliquoted for use in LAMP reactions for both onfarm and in-lab settings.

\section{Bacterial DNA isolation and quantification}

P. multocida, M. haemolytica, and H. somni were isolated and final DNA concentrations were quantified according to Mohan et al. [16]. Specifically, all pathogens were streaked on tryptic soy agar plates supplemented with defibrinated sheep blood (blood agar). P. multocida and M. haemolytica were incubated aerobically at $37{ }^{\circ} \mathrm{C}$ for $16-18 \mathrm{~h}$ while $\mathrm{H}$. somni was incubated in a $5 \% \mathrm{CO}_{2}$ atmosphere at $37^{\circ} \mathrm{C}$ for $2-3$ days by using BD GasPak ${ }^{\mathrm{TM}}$ EZ container systems (BD 260672) with $\mathrm{BD} \mathrm{BBL}^{\mathrm{TM}} \mathrm{CO}_{2}$ gas generators (BD 260679). Individual colonies of each bacterial species were picked from the blood agar plates. P. multocida and M. haemolytica were inoculated into brain-heart infusion (BHI) broth and $H$. somni was inoculated into tryptic soy broth (TSB). All were incubated in the same conditions as the plates.

DNA extraction of each pathogen was carried out by taking $2 \mathrm{~mL}$ of saturated liquid culture and following the PureLink $^{\mathrm{TM}}$ Genomic DNA Mini Kit (Catalog \#K182002, Invitrogen, Waltham, MA, USA) procedure. Final DNA concentrations $(\mathrm{ng} / \mu \mathrm{L})$ of eluted extracts were measured using the Quant-iT PicoGreen dsDNA Assay Kit (Invitrogen P11496).

\section{Colorimetric quantitative LAMP assay (qLAMP)}

The colorimetric assay was conducted by modifying the previously published procedure [16]. Specifically, in the colorimetric assay, the New England Biolabs' Warmstart Colorimetric LAMP $2 \times$ Master Mix was used. The mix was coupled with Antarctic Thermolabile 
uracil DNA glycosylase (UDG) and deoxyuridine triphosphate (dUTP) to minimize carryover contamination throughout the experiment. In-house validation experiments have confirmed that UDG/dUTP does not affect reaction performance at the concentration used. The LAMP solution comprised $12.5 \mu \mathrm{L}$ of this mix $\left(40 \mathrm{mM}\right.$ Tris- $\mathrm{HCl}, 20 \mathrm{mM}\left(\mathrm{NH}_{4}\right)_{2} \mathrm{SO}_{4}$, $100 \mathrm{mM} \mathrm{KCl}, 16 \mathrm{mM} \mathrm{MgSO}_{4}, 2.8 \mathrm{mM}$ dNTPs, $0.28 \mu \mathrm{M}$ dUTP, $0.64 \mathrm{U} / \mu \mathrm{L}$ Warmstart ${ }^{\circledR}$ Bst 2.0 DNA polymerase, $0.6 \mathrm{U} / \mu \mathrm{L}$ Warmstart ${ }^{\circledR}$ Reverse Transcriptase [RTx], $4 \times 10^{-4} \mathrm{U} / \mu \mathrm{L}$ Antarctic Thermolabile UDG, $200 \mathrm{mM}$ Phenol red, 0.2\% Tween 20, pH 8.8@25 ${ }^{\circ} \mathrm{C}$ ) (Catalog \# M1800L, New England Biolabs, Ipswich, MA, USA), $2.5 \mu \mathrm{L}$ of a $10 \times$ LAMP primer mixture $(10 \times$ concentration: $2 \mu \mathrm{M}$ F3, $2 \mu \mathrm{M}$ B3, $4 \mu \mathrm{M}$ LF, $4 \mu \mathrm{M}$ LB, $16 \mu \mathrm{M}$ FIP, $16 \mu \mathrm{M}$ BIP), $5 \mu \mathrm{L}$ of DNA-free water, and $5 \mu \mathrm{L}$ of DNA or mucus containing solution. Reactions were pipetted into wells of clear 96-well FrameStar ${ }^{\circledR}$ skirted flat optical bottom PCR plates (Catalog \#1149V67, Thomas Scientific, Swedesboro, NJ, USA). Wells were sealed with adhesive PCR plate seals (Catalog \#AB-0558, Thermo Fisher Scientific Waltham, MA, USA) and inserted into a CLARIOstar Plus (BMG Labtech Cary, NC, USA), a multi-mode plate reader with temperature control, for real-time colorimetric measurement. Spectra were collected from 350 to $750 \mathrm{~nm}$ with a step size of $5 \mathrm{~nm}$ for 60 cycles lasting approximately $60 \mathrm{~s}$ each. Reaction plates were incubated at $65{ }^{\circ} \mathrm{C}$ using the CLARIOstar Plus.

Each step in LAMP preparation (primer addition, template DNA loading, and reaction incubation/ measurement) was conducted in separate lab spaces in order to minimize false positives due to amplicon aerosol contamination. To further reduce contamination, RNase AWAY ${ }^{\mathrm{TM}}$ Surface Decontaminant (Thermo Fisher Scientific 14-754-34) was thoroughly applied to all working surfaces and lab gloves before and after use and wiped completely with Kimwipes to prevent residue formation.

\section{Data analysis}

Absorbance measurements for each minute at 430, 560, and $620 \mathrm{~nm}$ wavelengths were extracted, and the data were normalized using the formula (Equation 1):
The absorbance at $620 \mathrm{~nm}$ was used as a baseline, and the $430 \mathrm{~nm}$ and $520 \mathrm{~nm}$ wavelengths were used to mark the change in color of phenol red from red to yellow. The resulting ratios were plotted against time in Microsoft Excel.

\section{Colorimetric threshold}

A one-to-one mixture of $\mathrm{pH} 7.2$ phosphate-buffered saline (PBS, Thermo Fisher Scientific 20012050) and pH 8.5 Tris- $\mathrm{HCl}$ (Catalog \#SD8141, Bio Basic Amherst, NY, USA) was prepared. Using $0.1 \mathrm{M} \mathrm{NaOH}$ and $0.1 \mathrm{M}$ $\mathrm{HCl}$, the solution was adjusted to range between $\mathrm{pH}$ 6.0 and 8.0 with increments of approximately $0.2 \mathrm{pH}$ units. $5 \mu \mathrm{L}$ of the buffer solution were added to $12.5 \mu \mathrm{L}$ of Warmstart ${ }^{\circledR}$ Colorimetric LAMP $2 \times$ Master Mix and $7.5 \mu \mathrm{L}$ of DNA-free water. Each condition was added to a 96-well FrameStar ${ }^{\circledR}$ skirted flat optical bottom PCR plate in triplicate, sealed with a PCR film, and inserted into the CLARIOstar Plus to obtain measurements for three minutes. Data were analyzed as explained above and the values at each minute were averaged. From plotting the data, a colorimetric absorbance ratio was selected as the threshold according to the color changes observed so that colorimetric absorbance ratios above the threshold were considered positive and colorimetric absorbance ratios below the threshold were considered negative.

An image of the plate was taken using an Epson Perfection V800 Photo scanner (Catalog \#B11B223201, Amazon, Seattle, WA, USA) with settings set to professional mode, 48-bit color image type, and 720 dpi resolution. The image of the sample closest to the threshold value was processed using ImageJ to find the RGB values. Those RGB values were in turn used to calculate Hue Saturation Values (HSV) values. The Hue threshold was used to determine positive (above Hue threshold) versus negative (below Hue threshold) results in other assays.

\section{Primer screening and limit of detection (LOD)}

Colorimetric absorbance ratios were obtained from qLAMP experiments using a $2 \times$ DNA dilution factor (10 000-78.125 copies of DNA per reaction). Note that we are including the fraction of copies simply to indicate the dilution factor, the actual number of copies in a reaction would be rounded to the nearest whole number. All primer sets in Additional file 1 were tested and each

$$
\text { Colorimetric absorbance ratio }=\frac{\text { Absorbance at } 430 \mathrm{~nm}-\text { Absorbance at } 620 \mathrm{~nm}}{\text { Absorbance at } 520 \mathrm{~nm}-\text { Absorbance at } 620 \mathrm{~nm}}
$$


concentration of the template included three replicates for each primer set. DNA-free water was used as a control (no-template control, NTC).

Primer sets were scored by annotating the number of sufficient amplification reactions-defined as any replicate whose colorimetric absorbance ratio at $60 \mathrm{~min}$ was greater than 3.0-for each template concentration [including (NTC)] for each primer set. Any replicate that was deemed as sufficient amplification in the NTC was designated as a false positive. Any missing data for an entire template concentration was set at a constant value equal to the maximum colorimetric absorbance ratio observed across all primer sets at all concentrations. In contrast, any missing data for any given time point was filled with the value of the previous time point.

Primer sets were scored by first calculating the maximum colorimetric absorbance ratio and reaction time for each replicate at each template concentration (excluding NTC) for a given primer set. The average and standard deviation of these values were then calculated for each template concentration for a given primer set. Reaction time was defined as the first time point at which the absorbance ratio was greater than 3.0. For each primer set, the average of each one of these four metrics (average and standard deviation of maximum intensity and reaction time) was calculated across all template concentrations to assign a primer set metric (e.g., primer set average maximum colorimetric absorbance ratio). The LOD for each concentration was then calculated as the minimum template concentration where all replicates sufficiently amplify and all replicates of template concentrations above this minimum template concentration also sufficiently amplify. For cases where all replicates for all tested template concentrations amplified, the LOD was set at the lowest non-zero template concentration if there were less than three false positives. If all NTC reactions amplified (i.e., three false positives) or no replicates amplified at any template concentration, the LOD was set at -1 .

For the overall scoring of primer sets, ineligible primer sets (as designated by an LOD of -1 ) were automatically assigned an overall score of 0 and withdrawn from further scoring. All eligible primer sets were then assigned a weighted overall score, $S_{k}$, for a primer set, $k$, using the following expression:

$$
\begin{aligned}
S_{k}= & w_{\bar{I}} \cdot\left(1-\frac{\max (\bar{I})-I_{k}}{\operatorname{Range}(\bar{I})}\right) \\
& +\sum_{n} w_{n} \cdot\left(1-\frac{\min (n)-n_{k}}{\operatorname{Range}(n)}\right)
\end{aligned}
$$

$$
\begin{aligned}
& n \in\left(\sigma(I), \overline{t_{r x n}}, \sigma\left(t_{r x n}\right), L O D, F P\right) \\
& w_{n}=\left[\begin{array}{c}
35 \\
5 \\
30 \\
5 \\
5 \\
20
\end{array}\right] \text { for } \begin{array}{cc}
\bar{I} & \overline{t_{r x n}} \\
& \sigma\left(t_{r x n}\right) \\
& \text { LPD }
\end{array}
\end{aligned}
$$

where $\bar{I}, \sigma(I), \overline{t_{r x n}}, \sigma\left(t_{r x n}\right), L O D, F P$ is the set average maximum colorimetric absorbance ratio, set standard deviation of the maximum colorimetric absorbance ratio, set average reaction time, set standard deviation of the reaction time, set LOD, and the number of false positives for a given primer set, respectively. The range defined above is the maximum value minus the minimum value for a given set metric across all eligible primer sets. If the range for a given set metric was 0 (i.e., all primer sets had the same value), that set was given the full weight allotted for that set metric.

Primers with the highest scores were selected as the best primer sets to detect the bacteria of interest using a python script (Additional file 2). The worst LOD for the three selected primers was set as the LOD to be used in other experiments. Images of the plates were taken at 0 and 60 min using the Epson Perfection V800 Photo scanner.

\section{Combinatorial experiment with one, two, or three species spiked into water}

Colorimetric qLAMP assays were performed for $60 \mathrm{~min}$ using 1250 copies per reaction of gDNA of one, two, and/or three bacteria $P$. multocida, M. haemolytica, and $H$. somni in the same reaction. Each condition was repeated nine times in nine separate wells of 96-well plates. Images of the plates were taken at 0 and $60 \mathrm{~min}$ using the Epson Perfection V800 Photo scanner. Colorimetric absorbance ratios were calculated as explained above, and the resulting data were plotted against time. The finalized data were also analyzed in receiver operating characteristic (ROC) curves by using the colorimetric threshold previously determined and assessing positive versus negative reactions for each primer set. The highest number obtained from subtracting the false positive rate (Equation 2) from the true positive rate (Equation 3) was selected as the time threshold for that specific primer.

False positive rate (FPR)

$$
=\frac{\text { no. of false positives }}{\text { no. of false positives }+ \text { no. of true negatives }}
$$


True positive rate (TPR)

$$
=\frac{\text { no. of true positives }}{\text { no. of true positives }+ \text { no. of false negatives }}
$$

\section{Precision cooker experiments (on-farm and in-lab)}

LAMP reactions were prepared in individual domed PCR tubes (Thermo Fisher Scientific AB0337) using $12.5 \mu \mathrm{L}$ New England Biolabs' Warmstart ${ }^{\circledR}$ Colorimetric LAMP $2 \times$ Master Mix, $2.5 \mu \mathrm{L}$ of primer mix, $5 \mu \mathrm{L}$ of DNA free water, and $5 \mu \mathrm{L}$ of mucus sample. An Anova Culinary AN500-US00 Sous Vide Precision Cooker (Amazon B08CF6Y4WF) was filled with water and set to $149^{\circ} \mathrm{F}\left(65^{\circ} \mathrm{C}\right)$. The temperature of the water was verified in the lab using an Hti HT-04 Thermal Imaging Camera (Additional file 3). The tubes were submerged in the water on the right side (the region with a relatively homogenous temperature of $65{ }^{\circ} \mathrm{C}$ ) either by taping them to the inside of the precision cooker with heat-resistant 3/4-inch autoclave tape (Thermo Fisher Scientific 15904) or by using PCR tube holders designed and 3D-printed in-lab with a Formlabs Form 3B 3D printer (Formlabs, Somerville, MA, USA) using high-temperature resin v2 and $0.1 \mathrm{~mm}$ layer thickness (Additional files 4 and 5). The tubes were removed from the water after $60 \mathrm{~min}$.

The experiment was performed in-lab using the usual procedures to avoid contamination (RNase AWAY ${ }^{\mathrm{TM}}$ spray, separation of lab spaces, etc.) and on-farm. For the on-farm experiment, the reagents were prepared in the lab, and the addition of mucus was done on-farm using a $0.5-10 \mu \mathrm{L}$ single-channel pipette with no additional measures to avoid contamination (Additional file 6). The mucus addition on-farm happened no more than $30 \mathrm{~min}$ after extraction from the steers, while the mucus addition in the lab was done 4 days after collecting the samples (the samples being stored at $-80{ }^{\circ} \mathrm{C}$ in the meantime). The samples were stored in water so that the test matrix would be similar in the lab and on the farm.

Images of the tubes were taken at 0 and $60 \mathrm{~min}$. Images of the tubes in-lab were taken using the Epson Perfection V800 Photo scanner and images of tubes in-farm were taken using a Samsung Galaxy A50. All images obtained were adjusted by using the white balance tool on Adobe Lightroom to obtain a relatively uniform background. The RGB values of each solution were extracted at $60 \mathrm{~min}$ using ImageJ and Hue values were calculated to differentiate positive and negative results. Shadows and glows on the images were avoided during this process to increase the accuracy of the results. The Hue scale indicated on a color wheel from $0^{\circ}$ to $360^{\circ}$. Red/pink color is around $0-15^{\circ}$ and $345-360^{\circ}$, orange/yellow is around $30-60^{\circ}$.
Since we set a Hue value of 35 as cut-off (higher than 35 is a positive reaction), the red/pink color on the high end (close to $360^{\circ}$ ) was simply set to 0 to avoid confusion.

When comparing the LAMP farm results with PCR, having 2 out of 3 LAMP reactions show the same result as PCR was considered agreement.

\section{Results}

LAMP colorimetric performance

We evaluated the analytical sensitivity and specificity of primers designed to detect BRD pathogens as highlighted in Figure 1. A typical colorimetric response between positive and negative results is shown in Figure 2. We present the percent concordance between in-lab and onfarm LAMP as well as between PCR and on-farm LAMP using unprocessed mucus collected from steers (Table 1 and Additional file 7).

\section{Determining a threshold for colorimetric visualization}

LAMP reaction reagents were set to increasing $\mathrm{pH}$ to visualize color change (Figure 3 ). From the colorimetric results, 3.0 was selected as the threshold for colorimetric absorbance ratio. The color of the LAMP reaction at $\mathrm{pH}$ 6.63 (which is the closest to the 3.0 threshold of all the samples tested) was used to set the Hue threshold at 35 since at this point, the color change is distinctly different from the starting reaction color around $\mathrm{pH}$ 7.5-8.

\section{Down selection of primers for BRD detection through quantitative LAMP and LOD experiments}

From the primer screening carried out in Mohan et al. [16], the selected primers (Additional file 1) were narrowed down further to one per bacterial target. The optimal primers were selected based on calculated scores (Additional file 2) obtained from LOD colorimetric assays (Additional files 8, 9, 10). The primers with the highest scores were $\mathrm{kmt} 1$, rsmL, and lolB as highlighted in blue shaded data (Table 2). The worst limit of detection of the three selected primers, 1250 copies per reaction, was selected as the concentration used in later multiple isolate experiments.

Combinatorial experiment with one, two, or three bacterial species spiked in water using phenol red

Colorimetric qLAMP reactions with the selected primer sets were performed to determine assay performance with mixed bacterial samples. The previously determined limit of detection, 1250 copies per reaction of gDNA, was spiked in for each pathogen being tested in a reaction. Pathogens were tested in pairs and all together to simulate the bacterial communities present in actual mucus samples. The kmt1 (PM) and lolB (HS) primer sets were 


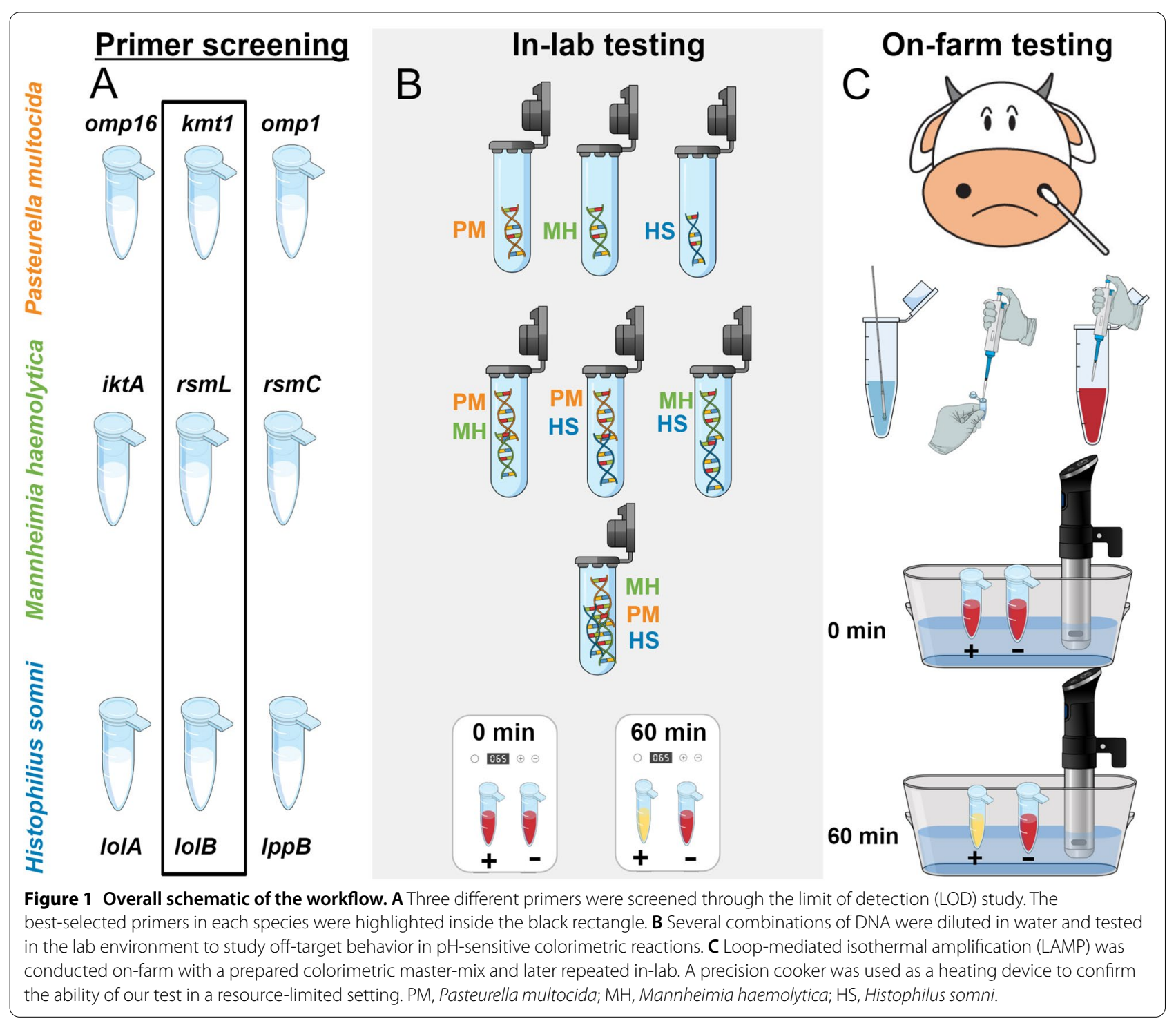

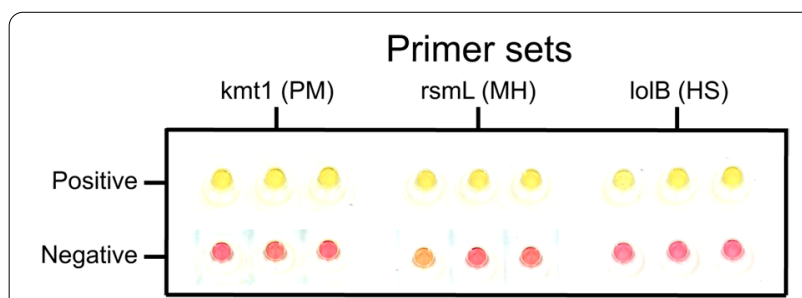

Figure 2 Representative colorimetric results for positive and negative reactions. Positives taken from quantitative loop-mediated isothermal amplification (qLAMP) reactions run with 10000 copies of DNA per reaction and negatives taken from qLAMP reactions without DNA. Kmt1 primers were used to detect PM, rsmL primers were used to detect $\mathrm{MH}$ and lolB primers were used to detect HS. All samples were imaged at $60 \mathrm{~min}$. Images were collected using an Epson Perfection V800 Photo scanner and the background was whitened using the ImageJ brightness/contrast setting. PM, Pasteurella multocida; MH, Mannheimia haemolytica; HS, Histophilus somni.
Table 1 Concordance between experiments in-lab and on-farm, and between the precision cooker assay on-farm and polymerase chain reaction (PCR)

\begin{tabular}{lll}
\hline Target pathogen & $\begin{array}{l}\text { \% Concordance: } \\
\text { precision cooker } \\
\text { on-farm vs. in-lab PCR } \\
\text { (\%) }\end{array}$ & $\begin{array}{l}\text { \% Concordance: } \\
\text { precision cooker } \\
\text { on-farm vs. in-lab (\%) }\end{array}$ \\
\hline P. multocida & 100.0 & 83.3 \\
M. haemolytica & 60.0 & 66.7 \\
H. somni & 100.0 & 66.7
\end{tabular}

Between loop-mediated isothermal amplification (LAMP) and PCR, 2 out of 3 LAMP reactions with the same result as PCR was considered agreement.

able to amplify the corresponding genes regardless of the bacterial combination and showed minimal false-positive results. RsmL (MH) also showed a colorimetric difference in the presence versus the absence of $M$. haemolytica 


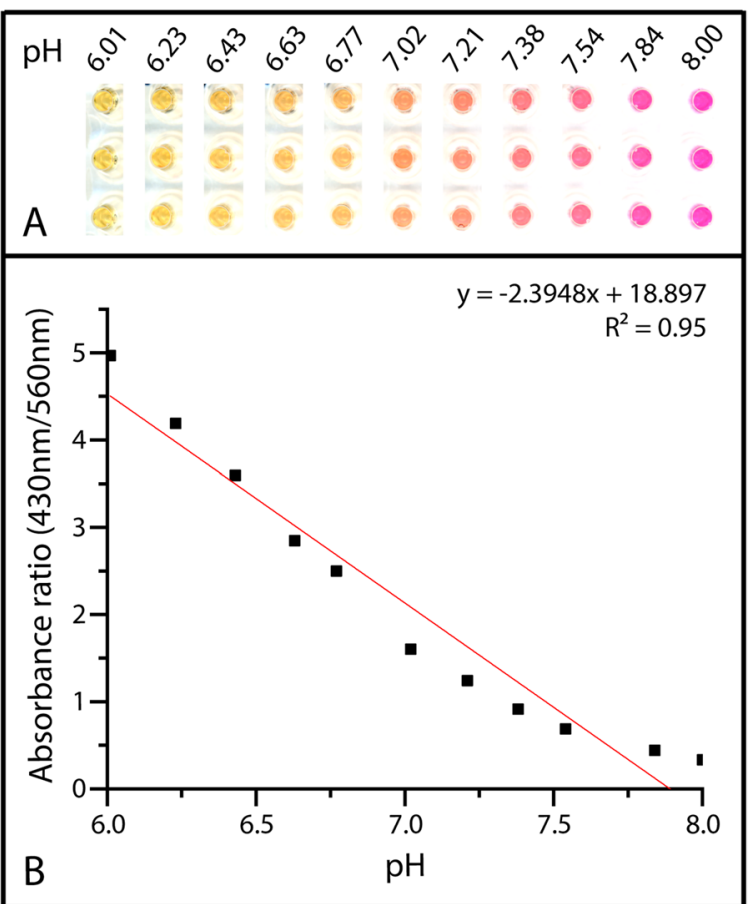

Figure 3 Colorimetric gradient at increasing $\mathrm{pH}$ values. Colorimetric detection of a 1:1 mixture of phosphate-buffered saline (PBS) and Tris-HCl buffer adjusted to $\mathrm{pH}$ 6.0-8.0 in approximately $0.2 \mathrm{pH}$ intervals. A Image collected using the Epson Perfection V800 Photo scanner and background whitened using the ImageJ brightness/contrast setting. B Average colorimetric absorbance ratios from three cycles collected with the CLARIOstar Plus. genes. However, it was not significant enough for the previously determined standards of 3.0 colorimetric absorbance threshold and 35 Hue value since some samples with target pathogenic DNA showed results below these values (Additional files 11 and 12).

These results were analyzed further by generating ROC curves for each primer set. Here, the kmt 1 and lolB primer sets show nearly perfect curves (analytical sensitivity of $100 \%(\mathrm{kmt})$ and $91.67 \%(\mathrm{lolB})$; analytical specificity of $100 \%(\mathrm{kmt} 1)$ and $100 \%(\mathrm{lolB}))$, while rsmL does not. This indicates that rsmL does not work well $(66.7 \%$ analytical sensitivity, $100 \%$ analytical specificity) and will need to be redesigned in future studies for improving analytical sensitivity (Figure 4). These curves were also used to identify a time threshold (i.e., if a reaction requires more than this threshold to obtain a colorimetric absorbance ratio of 3.0 , it is considered a negative result) for each of the primer sets by finding the time throughout the 60 -min period with the greatest difference between the true positive and false-positive rates. The selected time thresholds were: $41 \mathrm{~min}$ (kmt1), $59 \mathrm{~min}$ (rsmL), and $54 \mathrm{~min}$ (lolB) (Table 3).

\section{Colorimetric detection of BRD bacterial pathogens on-farm and in-lab}

The reagents for LAMP reactions were prepared in the lab and mucus from different steers was tested for the presence of $P$. multocida, M. haemolytica, and H. somni. The mucus was initially added on-farm after swabbing the corresponding steer and resuspending the swab contents in $200 \mu \mathrm{L}$ water. The reactions were then incubated

Table 2 Performance characteristics of all primer sets used for screening

\begin{tabular}{|c|c|c|c|c|c|c|c|c|}
\hline $\begin{array}{c}\text { Target } \\
\text { Pathogen }\end{array}$ & $\begin{array}{c}\text { Primer } \\
\text { Set }\end{array}$ & $\begin{array}{l}\text { LOD } \\
\text { (copies/ } \\
\text { rxn) }\end{array}$ & $\begin{array}{c}\text { Colorimetric } \\
\text { Absorbance } \\
\text { Ratio } \\
\text { Average }\end{array}$ & \begin{tabular}{|c|} 
Colorimetric \\
Absorbance \\
Ratio \\
Standard \\
Deviation \\
\end{tabular} & $\begin{array}{c}\text { Reaction } \\
\text { Time } \\
\text { Average } \\
\text { (min) }\end{array}$ & \begin{tabular}{|c|} 
Reaction \\
Time \\
Standard \\
Deviation \\
(min) \\
\end{tabular} & $\begin{array}{c}\text { False } \\
\text { Positives }\end{array}$ & $\begin{array}{l}\text { Overall } \\
\text { Score }\end{array}$ \\
\hline \multirow{3}{*}{ PM } & kmt1 & 78.13 & 5.71 & 0.33 & 29.58 & 2.44 & 0 & 99.46 \\
\hline & omp16 & -1 & 3.92 & 0.11 & 45.92 & 1.38 & 3 & 0 \\
\hline & ompP1 & 156.25 & 5.53 & 0.59 & 37.46 & 2.73 & 3 & 66.59 \\
\hline \multirow{3}{*}{$\mathrm{MH}$} & iktA & -1 & 5.41 & 0.47 & 36.63 & 2.58 & 3 & 0 \\
\hline & $\mathrm{rsmC}$ & 2500.00 & 2.70 & 0.85 & 55.63 & 3.41 & 0 & 25.88 \\
\hline & rsmL & 312.50 & 4.58 & 0.53 & 41.29 & 2.19 & 0 & 71.84 \\
\hline \multirow{3}{*}{ HS } & IolA & 625.00 & 3.09 & 0.39 & 50.29 & 2.05 & 0 & 44.4 \\
\hline & IOlB & 1250.00 & 5.36 & 1.49 & 38.71 & 5.69 & 0 & 72.93 \\
\hline & IppB & -1 & 2.94 & 1.00 & 53.58 & 4.19 & 0 & 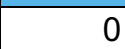 \\
\hline
\end{tabular}

Higher overall score indicates better performance.

PM, Pasteurella multocida; MH, Mannheimia haemolytica; HS, Histophilus somni.

The data highlighted in blue represents the selected primer sets as determined by the highest score for the target pathogen. 

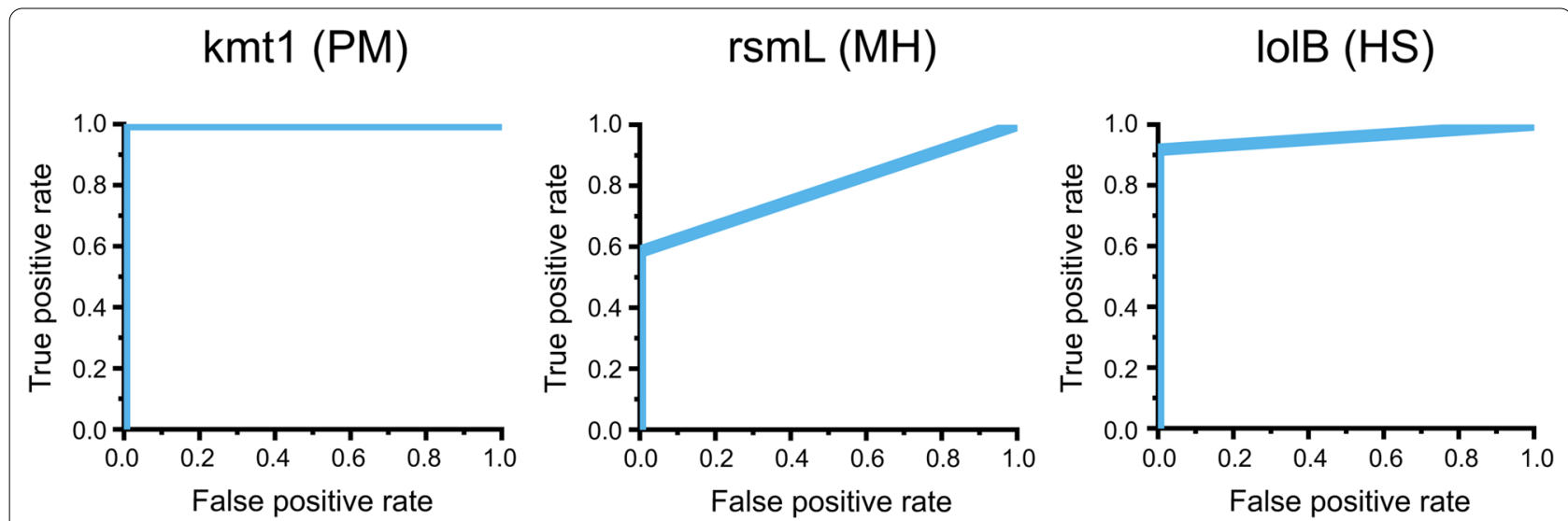

Figure 4 Receiver operating characteristic (ROC) curves for each selected primer set. The graphs illustrate the true-positive rate (TPR) and false-positive rate (FPR) of the quantitative loop-mediated isothermal amplification (qLAMP) assay for each primer using the combinations of bovine respiratory disease (BRD) bacteria presented in Additional files 11 and 12. TPR was calculated as TP/(TP + FN) and FPR was calculated as FP/(FP+TN). TP, true positive; FN, false negative; FP, false positive; TN, true negative; PM, Pasteurella multocida; MH, Mannheimia haemolytica; HS, Histophilus somni.

by being submerged in water at $65^{\circ} \mathrm{C}$ inside a precision cooker for 60 min (Figure 5). Four days later, the same assay was performed in a lab setting using the same mucus dilutions (Figure 6). The concordance observed between LAMP assays performed on the farm and in the lab varied between primers: kmt1 83.3\%, rsmL 66.7\%, lolB 66.7\%. Although lower than expected, in all the cases (except one) in which discordance was observed, the results were positive on-farm and negative in-lab. Therefore, the lack of consistency is likely due to the instability of the mucus samples (rather than the unreliability of the LAMP assay). These results further accentuate the necessity of field-based testing.

The mucus samples used were also analyzed in a PCR study. Within PCR, P. multocida was detected in all steers, $M$. haemolytica was only present in steers D and $\mathrm{E}$, and $H$. somni was detected in steers C, D, and E (Additional file 13). Of the 5 steers, 5 out of 5 of the ones tested for $P$. multocida and $H$. somni showed the same results between on-farm LAMP and PCR. In contrast, only 3 out of 5 of the ones tested for M. haemolytica were in agreement. From these results, we can conclude that LAMP is just as reliable as PCR in detecting the presence of pathogens as long as optimal primers are designed.

\section{Discussion}

Since the 2000s [12], LAMP has been a widely used method for the detection of pathogens, including bacterial [18-22] and viral targets [23-28]. While a majority of these efforts have been made using fluorescent dyes, there have been some advances in the detection of pathogens by coupling LAMP reagents with dyes that are sensitive to magnesium $[29,30]$ or $\mathrm{pH}[28,31-33]$. These dyes allow visualization of the result with the naked eye. In the assay presented here, we build on the primers previously designed by us [16] and couple them with a $\mathrm{pH}$-sensitive colorimetric reagent: phenol red [28]. The primers were screened through the LOD study. Their analytical sensitivity and specificity were determined by studying their behavior with on-target and off-target DNA mixtures, and the concordance of the reaction results between inlab and on-farm testing was analyzed (Figure 1).

As seen in previous studies, the Warmstart ${ }^{\circledR}$ LAMP $2 \times$ Master Mix, which contains phenol red, is characterized by its transition from pink to yellow as the LAMP reaction occurs and the $\mathrm{pH}$ decreases [28, 34]. Positive and negative results using our selected primers for the detection of P. multocida, M. haemolytica, H. somni, and the pink and yellow distinction can be observed in Figure 2.

While some work has been done to determine the presence of BRD pathogens [16, 35], there is no precedent showing that BRD pathogens can be accurately detected in a field setting. In this paper, Table 1 and Additional file 7 highlight the concordance between conducting the assays on-farm and in-lab. Surprisingly, there is a higher concordance between the on-farm LAMP and in-lab PCR, compared to on-farm and in-lab LAMP. This result suggests that the mucus transportation from the farm to the lab may lead to DNA degradation that leads to false negatives in LAMP reactions (but not in PCR).

In this work, we developed a colorimetric assay for BRD pathogens with the following six advantages: (i) it can be conducted on the farm using a simple consumergrade water bath, (ii) it provides a visual readout and thus, can be analyzed by the naked eye, (iii) it provides a response within $60 \mathrm{~min}$, (iv) it does not require sample processing (e.g., extraction of nucleic acids), (v) it can detect the pathogens $P$. multocida and H. somni with high 
Table 3 Loop-mediated isothermal amplification (LAMP) absorbance time thresholds with different combinations of PM, $\mathrm{MH}$, and HS gDNA

\begin{tabular}{|c|c|c|c|c|c|c|c|c|c|c|c|c|c|}
\hline $\begin{array}{l}\text { Time } \\
\text { threshold } \\
\text { (minutes) }\end{array}$ & Primers & PM & & & $\mathrm{MH}$ & & & HS & & & PM & MH & \\
\hline \multirow{3}{*}{41} & \multirow{3}{*}{ kmt1 } & 34 & 34 & 34 & 60 & 60 & 60 & 60 & 60 & 60 & 35 & 36 & 35 \\
\hline & & 38 & 34 & 35 & 60 & 60 & 60 & 60 & 60 & 60 & 37 & 36 & 36 \\
\hline & & 39 & 38 & 36 & 60 & 60 & 60 & 60 & 60 & 60 & 39 & 37 & 38 \\
\hline \multirow{3}{*}{59} & \multirow{3}{*}{ rsmL } & 60 & 60 & 60 & 56 & 42 & 45 & 60 & 60 & 60 & 60 & 44 & 43 \\
\hline & & 60 & 60 & 60 & 59 & 50 & 56 & 60 & 60 & 60 & 60 & 49 & 44 \\
\hline & & 60 & 60 & 60 & 60 & 51 & 60 & 60 & 60 & 60 & 60 & 59 & 49 \\
\hline \multirow{3}{*}{54} & \multirow{3}{*}{ lolB } & 60 & 60 & 60 & 60 & 60 & 60 & 37 & 39 & 41 & 60 & 60 & 60 \\
\hline & & 60 & 60 & 60 & 60 & 60 & 60 & 41 & 39 & 53 & 60 & 60 & 60 \\
\hline & & 60 & 60 & 60 & 60 & 60 & 60 & 46 & 40 & 60 & 60 & 60 & 60 \\
\hline
\end{tabular}

\begin{tabular}{|c|c|c|c|c|c|c|c|c|c|c|c|c|c|}
\hline $\begin{array}{l}\text { Time } \\
\text { threshold } \\
\text { (minutes) }\end{array}$ & Primers & \multicolumn{3}{|c|}{$\mathrm{PM}+\mathrm{HS}$} & \multicolumn{3}{|c|}{$\mathrm{MH}+\mathrm{HS}$} & \multicolumn{3}{|c|}{$\mathrm{PM}+\mathrm{MH}+\mathrm{HS}$} & \multicolumn{3}{|c|}{ NTC } \\
\hline \multirow{3}{*}{41} & \multirow{3}{*}{ kmt1 } & 34 & 36 & 37 & 60 & 60 & 60 & 36 & 36 & 36 & 60 & 60 & 60 \\
\hline & & 35 & 36 & 40 & 60 & 60 & 60 & 37 & 38 & 37 & 60 & 60 & 60 \\
\hline & & 39 & 37 & 41 & 60 & 60 & 60 & 39 & 38 & 37 & 60 & 60 & 60 \\
\hline \multirow{3}{*}{59} & \multirow{3}{*}{$\mathrm{rsmL}$} & 60 & 60 & 60 & 60 & 55 & 43 & 60 & 44 & 42 & 60 & 60 & 60 \\
\hline & & 60 & 60 & 60 & 60 & 60 & 45 & 60 & 58 & 45 & 60 & 60 & 60 \\
\hline & & 60 & 60 & 60 & 60 & 60 & 60 & 60 & 60 & 54 & 60 & 60 & 60 \\
\hline \multirow{3}{*}{54} & \multirow{3}{*}{ lolB } & 37 & 42 & 36 & 40 & 38 & 36 & 42 & 36 & 38 & 60 & 60 & 60 \\
\hline & & 46 & 44 & 38 & 40 & 50 & 45 & 44 & 36 & 40 & 60 & 60 & 60 \\
\hline & & 52 & 60 & 43 & 42 & 60 & 54 & 45 & 36 & 44 & 60 & 60 & 60 \\
\hline
\end{tabular}

Data illustrating the time (in minutes) obtained from Figure 4 and the samples in which the colorimetric absorbance threshold $(430 \mathrm{~nm} / 520 \mathrm{~nm}$ ) of 3.0 was crossed for each combination of Pasteurella multocida (PM), Mannheimia haemolytica (MH), and Histophilus somni (HS) in the quantitative LAMP (qLAMP) assay shown in Additional file 12. Primer set kmt1 detects PM, rsmL detects MH and lolB detects HS. A value of 60 indicates that the colorimetric absorbance ratio did not reach the 3.0 threshold. The values highlighted in blue are less than or equal to the time threshold for the respective primer set and hence considered positive amplification.

accuracy (100\% and 96\%, respectively), and (vi) it utilizes a simple non-invasive nasal swab for sampling.

A major limitation of the current assay is the poor performance of the rsmL primer set for targeting M. haemolytica. Even though we performed several screening steps first in our previous work [16] and then in the current work, the primer set had poor performance (accuracy of 79\%) mainly due to false negatives. Since the primer set was performing well in the pure $M$. haemolytica sample, we speculate that the drop in performance is due to cross-reactivity with other off-target DNA. We will redesign the primer sets for targeting $M$. haemolytica in future work. Another limitation is the low number of clinical samples tested. Although these numbers are sufficient to demonstrate the feasibility of on-farm visual LAMP, they are not sufficient to demonstrate clinical performance. This study serves as a building block for future larger-scale studies.

We anticipate that due to the simple nature of the assay, it can be coupled to the visual observation of animals for clinical signs and help assess the cause of BRD. The assay can determine whether the P. multocida and $H$. somni are present in the animals displaying symptoms. The focus of the current work was on demonstrating the feasibility of conducting a visual molecular assay on the farm (instead of the lab). Only the detection of BRD pathogens is insufficient for clinical diagnosis in BRD since these pathogens could also be present in healthy animals; thus, we did not evaluate diagnostic specificity, sensitivity, and accuracy. 


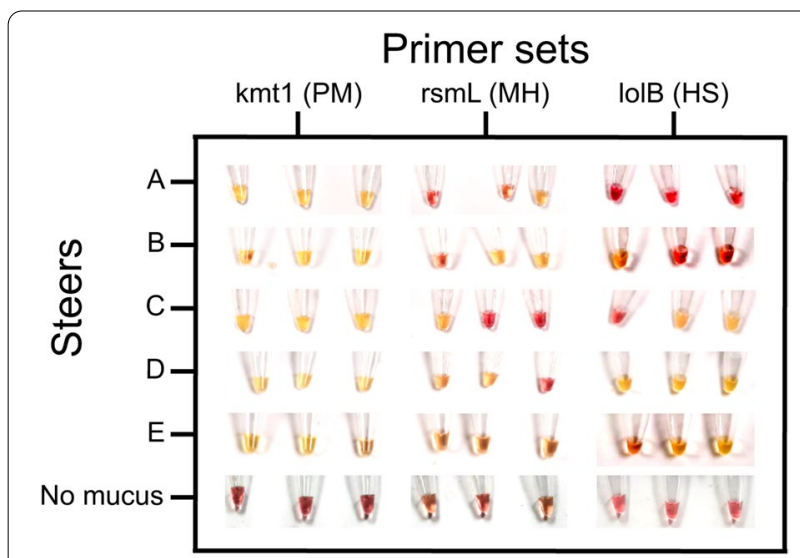

Figure 5 Colorimetric results of on-farm loop-mediated isothermal amplification (LAMP) detection of bacteria in unprocessed mucus collected from steers. LAMP reactions were run in polymerase chain reaction (PCR) tubes submerged in water at $65^{\circ} \mathrm{C}$ inside a precision cooker. kmt1 detects $\mathrm{PM}$, rsmL detects $\mathrm{MH}$ and lolB detects $\mathrm{HS}$. The reactions were run for $60 \mathrm{~min}$. DNA-free water was used as a negative control. Reactions were diluted in water. Images were collected with a Samsung Galaxy A50 and adjusted using the brightness/contrast tool on ImageJ and the white balance tool on Adobe Lightroom. See Additional file 13 for associated PCR results. PM, Pasteurella multocida; $\mathrm{MH}$, Mannheimia haemolytica; HS, Histophilus somni.

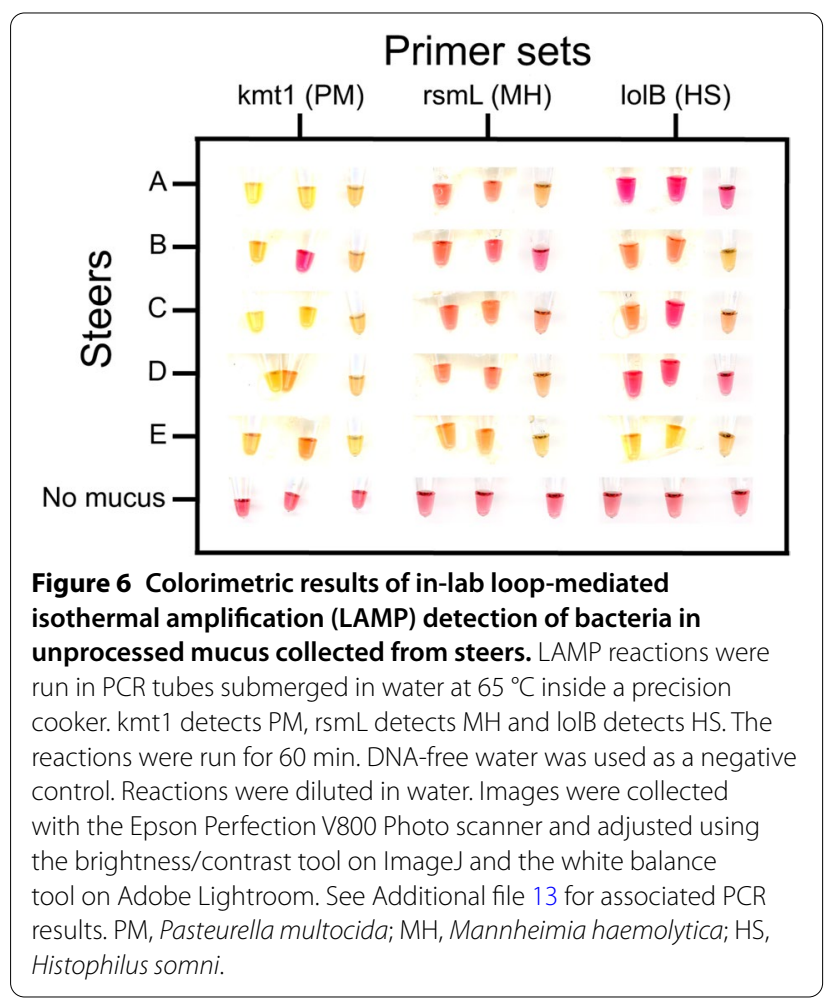

With further development, quantification of these BRD pathogens could help distinguish between healthy and sick animals. Once we include more targets (e.g., Mycoplasma bovis, viruses, antimicrobial resistance genes) in our assay, it could also help guide the treatment regimen for BRD.

\section{Abbreviations}

ADDL: animal disease diagnostic laboratory; BHI: brain-heart infusion; BRD: bovine respiratory disease; dUTP: deoxyuridine triphosphate; ELISA: enzymelinked immunosorbent assays; FN: false negative; FP: false positive; FPR: false positive rate; HS: Histophilus somni; HSV: hue saturation values; LAMP: loopmediated isothermal amplification; LOD: limit of detection; MH: Mannheimia haemolytica; NTC: no-template control; PBS: phosphate buffered saline; PCR: polymerase chain reaction; PM: Pasteurella multocida; qLAMP: quantitative loop-mediated isothermal amplification; ROC: receiver operating characteristic; TN: true negative; TP: true positive; TPR: true positive rate; TSB: tryptic soy broth; UDG: uracil DNA glycosylase.

\section{Supplementary Information}

The online version contains supplementary material available at https://doi. org/10.1186/s13567-021-00997-9.

\section{Additional file 1. Screened primer set sequences developed in [16].}

Additional file 2. LOD analysis script. The attached python script, ColorimetricAnalysis.py, was used to analyze primer set screening data. To execute, simply import the file and execute "getPrimerSummer(filename, output)" and provide the file name or path along with the output.xls file name or path. The resulting Excel file will contain two sheets; one containing the final primer scoring and the other containing intermediate calculations for each concentration in each primer set.

Additional file 3. A top-down thermal Image of precision cooker used for LAMP water bath experiments. Thermal image of the precision cooker used to heat LAMP reactions. The red cursor indicates the point of the highest temperature $\left(65.3^{\circ} \mathrm{C}\right)$ and corresponds to the color white. The green cursor indicates the point of the lowest temperature $\left(23.6^{\circ} \mathrm{C}\right)$ and corresponds to the color black, which is outside the boundaries of the pressure cooker. The central point is indicated by the white cursor and is $65.2^{\circ} \mathrm{C}$ ). LAMP reactions were submerged in the water on the right side of the precision cooker

Additional file 4. 3D model of the PCR tubes holder. A. PCR tube holder with 2 hanging parts for convenient placement of the tubes and three sets of eight tubes each. B. Slider to cover the tubes from floating in the precision cooker.

Additional file 5. 3D model of the PCR tubes holder.stl files. A. PCR tube holder.stl file. B. Slider to cover the tubes.stl file. Units are $\mathrm{mm}$.

Additional file 6. LAMP procedure on the farm. A. Nasal sample was extracted from a steer. B. The extracted mucus on the swab was diluted to $200 \mu \mathrm{L}$ of water. C. $5 \mu \mathrm{L}$ of resuspended nasal swab solution was used as a sample. D. $5 \mu \mathrm{L}$ of resuspended nasal swab solution were added to pre-prepared colorimetric LAMP tubes with different primer sets. $\mathbf{b}$. The tubes were incubated for $60 \mathrm{~min}$ at $65^{\circ} \mathrm{C}$ inside the precision cooker. Previous experiments ensured the submersion of PCR tubes would not cause inward leaking.

Additional file 7. On-farm vs. in-lab Hue values. Hue values from the precision cooker experiment on-farm shown in Figure 5 and in-lab shown in Figure 6. For both LAMP experiments and PCR, cells in blue indicate positive samples and cells in white indicate negative samples. Between farm LAMP and $P C R, 2$ out of 3 LAMP reactions with the same result as PCR was considered agreement.

Additional file 8. Limit of detection absorbance values. Limit of detection table showing selection process for primers for each gene target. The numbers 
indicate the colorimetric absorbance ratio of absorbance measured at $430 \mathrm{~nm}$ to $520 \mathrm{~nm}$. Blue cells correspond to colorimetric absorbance ratios higher than 3.0. Missing data indicates conditions that were not tested.

Additional file 9. LAMP colorimetric results with $\mathrm{PM}, \mathrm{MH}$, and $\mathrm{HS}$ gDNA present at $60 \mathrm{~min}$. Water-suspended DNA extracts of the corresponding gDNA were added to water to generate two-fold serial dilutions (10 000 to 78.125 copies of DNA/reaction). $\mathrm{kmt} 1$ detects PM, rsmL detects $\mathrm{MH}$ and lolB detects HS. These were added to qLAMP assays with the primer sets being tested for $60 \mathrm{~min}$ at $65^{\circ} \mathrm{C}$. Water was used as a negative control. Images were collected using the Epson Perfection V800 Photo scanner, and the background was whitened using the ImageJ brightness/contrast setting. PM: Pasteurella multocida, MH: Mannheimia haemolytica, HS: Histophilus somni.

Additional file 10. Quantitative results of PM, MH, and HS gDNA present in water. Water-suspended DNA extracts of the corresponding gDNA were added to water to generate two-fold serial dilutions (10 000 to 78.125 copies of DNA/reaction). $\mathrm{kmt} 1$ detects $\mathrm{PM}$, rsmL detects $\mathrm{MH}$ and lolB detects $\mathrm{HS}$. These were added to qLAMP assays with the primer sets being tested for $60 \mathrm{~min}$ at $65^{\circ} \mathrm{C}$. Absorbance ratios above 3.0 were considered positive and absorbance ratios below 3.0 were considered negative. Water was used as a negative control. For primers $\mathrm{lo} \mid \mathrm{A}$, lolB and $\mathrm{lppB}$ the data points at minute 59 were excluded due to them being negative values potentially due to instrument error. Each panel has three replicates. PM: Pasteurella multocida, MH: Mannheimia haemoIytica, HS: Histophilus somni.

Additional file 11. LAMP colorimetric results with different combinations of PM, MH, and $\mathrm{HS}$ gDNA present in water at $\mathbf{6 0} \mathrm{min}$. Water-suspended DNA extracts at 1,250 copies of DNA per reaction were added to qLAMP assays with the primer sets being tested for $60 \mathrm{~min}$ at $65^{\circ} \mathrm{C}$. kmt1 detects PM, rsmL detects $\mathrm{MH}$ and lolB detects HS. DNA-free water was used as a negative control. Images were collected using the Epson Perfection V800 Photo scanner, and the background was whitened using the ImageJ brightness/contrast setting. PM: Pasteurella multocida, MH: Mannheimia haemolytica, HS: Histophilus somni.

Additional file 12. Quantitative results of LAMP detection of different combinations of PM, MH, and HS gDNA present in water at $60 \mathrm{~min}$. Watersuspended DNA extracts at 1250 copies of DNA per reaction were added to qLAMP assays, with the primer sets being tested for $60 \mathrm{~min}$ at $65^{\circ} \mathrm{C}$. kmt1 detects PM, rsmL detects $\mathrm{MH}$ and lolB detects HS. Absorbance ratios above 3.0 were considered positive and absorbance ratios below 3.0 were considered negative. DNA-free water was used as a negative control. Each panel has nine replicates. PM: Pasteurella multocida, $\mathrm{MH}$ : Mannheimia haemolytica, $\mathrm{HS}$ : Histophilus somni.

Additional file 13. PCR confirmation for 5 steers with 3 different primers corresponding to PM, MH, and HS. PCR was conducted with the extracted genomic DNA from the mucus obtained from respective steers. PCR was performed using Thermo Fisher Phusion ${ }^{\mathrm{TM}}$ High-Fidelity DNA Polymerase (F-530XL). The extracted genomic DNA was used as a template for the PCR reaction. $1 \%$ agarose gel was used to run the PCR product with $1 \mathrm{kbp}$ DNA ladder (NEB N0468S) as a marker. Expected gene sizes were PM: ompP1 - 1180 bp MH: iktA - 1932 bp and HS: IppB - 404 bp. PM: Pasteurella multocida, MH: Mannheimia haemolytica, HS: Histophilus somni.

\section{Acknowledgements}

We would like to acknowledge Rohit Verma for inspiring the use of a sous vide precision cooker as a water bath heat source.

\section{Authors' contributions}

AP-G designed and performed experiments, analyzed data, wrote, and revised the manuscript. MKM and JLD made substantial contributions to data acquisition. AA and JPS arranged sample collection. GR designed and printed the 3D model. DP provided bacterial isolates and culture conditions. JK provided training on sampling. TJ provided guidance on gene targets. MSV coordinated the entire project, revised the manuscript, and made substantial contributions to experimental design. All authors read and approved the final manuscript.

\section{Funding}

This work is supported by the Agriculture and Food Research Initiative Competitive Grants Program Award 2020-68014-31302 from the U.S. Department of Agriculture National Institute of Food and Agriculture. This work is also supported by Purdue University's Colleges of Agriculture and Engineering Collaborative Projects Program 2018, the College of Agriculture and Wabash Heartland Innovation Network Graduate Student Support program, an Agricultural Science and Extension for Economic Development (AgSEED) Grant, and the Disease Diagnostics INventors Challenge (created by the Purdue Institute of Inflammation, Immunology and Infectious Disease in partnership with the Department of Comparative Pathobiology, which contributed the funds to realize the project, the Indiana Clinical and Translational Sciences Institute, and the Indiana Consortium for Analytical Science and Engineering).

\section{Availability of data and materials}

The data supporting the conclusions of this article are included within the article and its additional files.

\section{Declarations}

Ethics approval and consent to participate

The experiments were approved by the Purdue Animal Care and Use Committee (Approval Number 1906001911).

\section{Consent for publication}

Written informed consent for publication of their images was obtained from the authors conducting the assay. A copy of the consent form is available for review by the Editor of this journal.

\section{Competing interests}

Mohit S. Verma has interests in Krishi Inc., a company interested in licensing the technology developed here. The work performed here was not funded by Krishi Inc.

\section{Author details}

${ }^{1}$ Department of Agricultural and Biological Engineering, Purdue University, 225 S University Street, West Lafayette, IN 47907, USA. ${ }^{2}$ Department of Biochemistry, Purdue University, 175 South University Street, West Lafayette, IN 47906, USA. ${ }^{3}$ Birck Nanotechnology Center, Purdue University, 1205 W State St, West Lafayette, IN 47907, USA. ${ }^{4}$ School of Electrical and Computer Engineering, Purdue University, 465 Northwestern Avenue, West Lafayette, IN 47907, USA. ${ }^{5}$ Weldon School of Biomedical Engineering, Purdue University, 206 South Martin Jischke Drive, West Lafayette, IN 47907, USA. ${ }^{6}$ Department of Comparative Pathobiology, Purdue University, 625 Harrison Street, West Lafayette, IN 47907, USA. ${ }^{7}$ School of Veterinary Medicine, Texas Tech University, 7671 Evans Drive, Amarillo, TX 79106, USA. ${ }^{8}$ Department of Animal Sciences, Purdue University, 270 S Russell Street, West Lafayette, IN 47907, USA.

Received: 4 June 2021 Accepted: 3 September 2021

Published online: 02 October 2021

\section{References}

1. USDA (2013) Feedlot 2011 Part IV: health and health management on U.S. feedlots with a capacity of 1,000 or more head

2. Wilson BK, Richards CJ, Step DL, Krehbiel CR (2017) Best management practices for newly weaned calves for improved health and well-being. Anim Sci J 95:2170-2182. https://doi.org/10.2527/jas.2016.1006

3. Johnson KK, Pendell DL (2017) Market impacts of reducing the prevalence of bovine respiratory disease in United States beef cattle feedlots. Front Vet Sci 4:189. https://doi.org/10.3389/fvets.2017.00189

4. Griffin D, Chengappa MM, Kuszak J, McVey DS (2010) Bacterial pathogens of the bovine respiratory disease complex. Vet Clin North Am Food Anim Pract 26:381-394. https://doi.org/10.1016/j.cvfa.2010.04.004

5. Taylor JD, Fulton RW, Lehenbauer TW, Step DL, Confer AW (2010) The epidemiology of bovine respiratory disease: what is the evidence for predisposing factors? Can Vet J 51:1095-1102

6. Klima CL, Holman DB, Ralston BJ, Stanford K, Zaheer R, Alexander TW, McAllister TA (2019) Lower respiratory tract microbiome and resistome of bovine respiratory disease mortalities. Microb Ecol 78:446-456. https:// doi.org/10.1007/s00248-019-01361-3 
7. Fulton RW, Confer AW (2012) Laboratory test descriptions for bovine respiratory disease diagnosis and their strengths and weaknesses: gold standards for diagnosis, do they exist? Can Vet J 53:754-761

8. Pansri P, Katholm J, Krogh KM, Aagaard AK, Schmidt LMB, Kudirkiene E, Larsen LE, Olsen JE (2020) Evaluation of novel multiplex qPCR assays for diagnosis of pathogens associated with the bovine respiratory disease complex. Vet J 256:105425. https://doi.org/10.1016/j.tvjl.2020.105425

9. Avra TD, Abell KM, Shane DD, Theurer ME, Larson RL, White BJ (2017) A retrospective analysis of risk factors associated with bovine respiratory disease treatment failure in feedlot cattle. J Anim Sci 95:1521-1527. https://doi.org/10.2527/jas.2016.1254

10. CDC (2013) Antibiotic resistance threats in the United States, 2013

11. Piepenburg O, Williams $C H$, Stemple DL, Armes NA (2006) DNA detection using recombination proteins. PLoS Biol 4:e204. https://doi.org/10.1371/ journal.pbio.0040204

12. Notomi T, Okayama H, Masubuchi H, Yonekawa T, Watanabe K, Amino N, Hase T (2000) Loop-mediated isothermal amplification of DNA. Nucleic Acids Res 28:e63. https://doi.org/10.1093/nar/28.12.e63

13. Nzelu CO, Kato H, Peters NC (2019) Loop-mediated isothermal amplification (LAMP): an advanced molecular point-of-care technique for the detection of Leishmania infection. PLoS Negl Trop Dis 13:e0007698. https://doi.org/10.1371/journal.pntd.0007698

14. Sirichaisinthop J, Buates S, Watanabe R, Han E-T, Suktawonjaroenpon W, Krasaesub S, Takeo S, Tsuboi T, Sattabongkot J (2011) Evaluation of loopmediated isothermal amplification (LAMP) for malaria diagnosis in a field setting. Am J Trop Med Hyg 85:594-596. https://doi.org/10.4269/ajtmh. 2011.10-0676

15. Conrad CC, Daher RK, Stanford K, Amoako KK, Boissinot M, Bergeron MG, Alexander T, Cook S, Ralston B, Zaheer R, Niu YD, McAllister T (2020) A sensitive and accurate recombinase polymerase amplification assay for detection of the primary bacterial pathogens causing bovine respiratory disease. Front Vet Sci 7:208. https://doi.org/10.3389/fvets.2020.00208

16. Mohan S, Pascual-Garrigos A, Brouwer H, Pillai D, Koziol J, Ault A, Schoonmaker J, Johnson T, Verma MS (2021) Loop-mediated isothermal amplification for the detection of Pasteurella multocida, Mannheimia haemolytica, and Histophilus somni in bovine nasal samples. ACS Agric Sci Technol 1:100-108. https://doi.org/10.1021/acsagscitech.0c00072

17. Kozel TR, Burnham-Marusich AR (2017) Point-of-care testing for infectious diseases: past, present, and future. J Clin Microbiol 55:2313-2320. https:// doi.org/10.1128/JCM.00476-17

18. Misawa Y, Yoshida A, Saito R, Yoshida H, Okuzumi K, Ito N, Okada M, Moriya K, Koike K (2007) Application of loop-mediated isothermal amplification technique to rapid and direct detection of methicillin-resistant Staphylococcus aureus (MRSA) in blood cultures. J Infect Chemother 13:134-140. https://doi.org/10.1007/s10156-007-0508-9

19. Wang L, Li Y, Chu J, Xu Z, Zhong Q (2012) Development and application of a simple loop-mediated isothermal amplification method on rapid detection of Listeria monocytogenes strains. Mol Biol Rep 39:445-449. https://doi.org/10.1007/s11033-011-0757-7

20. Xu Z, Li L, Chu J, Peters BM, Harris ML, Li B, Shi L, Shirtliff ME (2012) Development and application of loop-mediated isothermal amplification assays on rapid detection of various types of staphylococci strains. Food Res Int 47:166-173. https://doi.org/10.1016/j.foodres.2011.04.042

21. Zhao X, Wang L, Chu J, Li Y, Li Y, Xu Z, Li L, Shirtliff ME, He X, Liu Y, Wang J, Yang $L$ (2010) Development and application of a rapid and simple loopmediated isothermal amplification method for food-borne Salmonella detection. Food Sci Biotechnol 19:1655-1659. https://doi.org/10.1007/ s10068-010-0234-4

22. Zhao X, Li Y, Wang L, You L, Xu Z, Li L, He X, Liu Y, Wang J, Yang L (2010) Development and application of a loop-mediated isothermal amplification method on rapid detection Escherichia coli 0157 strains from food samples. Mol Biol Rep 37:2183-2188. https://doi.org/10.1007/ s11033-009-9700-6
23. Blomström A-L, Hakhverdyan M, Reid SM, Dukes JP, King DP, Belák S, Berg M (2008) A one-step reverse transcriptase loop-mediated isothermal amplification assay for simple and rapid detection of swine vesicular disease virus. J Virol Methods 147:188-193. https://doi.org/10.1016/j.jviro met.2007.08.023

24. Dukes JP, King DP, Alexandersen S (2006) Novel reverse transcription loop-mediated isothermal amplification for rapid detection of foot-andmouth disease virus. Arch Virol 151:1093-1106. https://doi.org/10.1007/ s00705-005-0708-5

25. Hagiwara M, Sasaki H, Matsuo K, Honda M, Kawase M, Nakagawa H (2007) Loop-mediated isothermal amplification method for detection of human papillomavirus type 6, 11, 16, and 18. J Med Virol 79:605-615. https://doi. org/10.1002/jmv.20858

26. Hong TCT, Mai QL, Cuong DV, Parida M, Minekawa H, Notomi T, Hasebe F, Morita K (2004) Development and evaluation of a novel loop-mediated isothermal amplification method for rapid detection of severe acute respiratory syndrome coronavirus. J Clin Microbiol 42:1956-1961. https:// doi.org/10.1128/jcm.42.5.1956-1961.2004

27. Okamoto S, Yoshikawa T, Ihira M, Suzuki K, Shimokata K, Nishiyama Y, Asano Y (2004) Rapid detection of varicella-zoster virus infection by a loop-mediated isothermal amplification method. J Med Virol 74:677-682. https://doi.org/10.1002/jmv.20223

28. Davidson JL, Wang J, Maruthamuthu MK, Dextre A, Pascual-Garrigos A, Mohan S, Putikam SVS, Osman FOI, McChesney D, Seville J, Verma MS (2021) A paper-based colorimetric molecular test for SARS-CoV-2 in saliva. Biosens Bioelectron X 9:100076. https://doi.org/10.1016/j.biosx.2021. 100076

29. Choopara I, Teethaisong Y, Arunrut N, Thunyaharn S, Kiatpathomchai W, Somboonna N (2021) Specific and sensitive, ready-to-use universal fungi detection by visual color using ITS1 loop-mediated isothermal amplification combined hydroxynaphthol blue. PeerJ 9:e11082. https://doi.org/10. 7717/peerj.11082

30. Nguyen DV, Nguyen VH, Seo TS (2019) Quantification of colorimetric loop-mediated isothermal amplification process. BioChip J 13:158-164. https://doi.org/10.1007/s13206-019-3206-7

31. Poole CB, Li Z, Alhassan A, Guelig D, Diesburg S, Tanner NA, Zhang Y, Evans TC, LaBarre P, Wanji S, Burton RA, Carlow CKS (2017) Colorimetric tests for diagnosis of filarial infection and vector surveillance using non-instrumented nucleic acid loop-mediated isothermal amplification (NINA-LAMP). PLoS One 12:e0169011. https://doi.org/10.1371/journal. pone.0169011

32. Tanner NA, Zhang Y, Evans TC (2015) Visual detection of isothermal nucleic acid amplification using $\mathrm{pH}$-sensitive dyes. Biotechniques 58:59-68. https://doi.org/10.2144/000114253

33. Wu S, Liu X, Ye S, Liu J, Zheng W, Dong X, Yin X (2021) Colorimetric isothermal nucleic acid detection of SARS-CoV-2 with dye combination. Heliyon 7:e06886. https://doi.org/10.1016/j.heliyon.2021.e06886

34. Peltzer D, Tobler K, Fraefel C, Maley M, Bachofen C (2021) Rapid and simple colorimetric loop-mediated isothermal amplification (LAMP) assay for the detection of Bovine alphaherpesvirus 1. J Virol Methods 289:114041. https://doi.org/10.1016/j.jviromet.2020.114041

35. Moustafa AM, Bennett MD (2017) Development of loop-mediated isothermal amplification-based diagnostic assays for detection of Pasteurella multocida and hemorrhagic septicemia-associated $P$ multocida serotype B:2. Am J Vet Res 78:134-143. https://doi.org/10.2460/ajvr.78.2.134

\section{Publisher's Note}

Springer Nature remains neutral with regard to jurisdictional claims in published maps and institutional affiliations. 